\title{
Leveraging Big Data Analytics to Improve Quality of Care in Health Care: A fsQCA Approach
}

\author{
Yichuan Wang \\ Newcastle University, United Kingdom \\ yi-chuan.wang@newcastle.ac.uk
}

\begin{abstract}
Academics across disciplines such as information systems, computer science and healthcare informatics highlight that big data analytics (BDA) have the potential to provide tremendous benefits for healthcare industries. Nevertheless, healthcare organizations continue to struggle to make progress on their $B D A$ initiatives. Drawing on the configuration theory, this paper proposes a conceptual framework to explore the impact of $B D A$ on improving quality of care in health care. Specifically, we investigate how BDA capabilities interact with complementary organizational resources and organizational capabilities in multiple configurations to achieve higher quality of care. Fuzzy-set qualitative comparative analysis ( $\left.f_{S} Q C A\right)$, which is a relatively new approach, was employed to identify five different configurations that lead to higher quality of care. These findings offer evidence to suggest that a range of solutions leading to better healthcare performance can indeed be identified through the effective use of $B D A$ and other organizational elements.
\end{abstract}

\section{Introduction}

Big data analytics (BDA) is increasingly being advocated as an important strategic information technology (IT) investment for healthcare organizations. Although building a big data analytical solution is costly, BDA has the potential to harvest data-driven insights, support evidence-based medicine, and improve quality of care at a lower cost [1], [2].

A few studies explore the impact of BDA on organizational performance through the resource-based view, knowledge based view, information processing view, and dynamic capability view (e.g. [3], [4], [5], [6]). From an information processing view, researchers explore the compelling pathways starting from analytics use capabilities, through insights and decisions, to organizational benefits over time (e.g. [4], [7]). Drawing on the resource-based view, some studies identify critical success factors of big data analytics such as big data analytics infrastructure and functionalities [3] [8] [9] [10], analytical people [4] [5], data-driven decision-marking culture [4] and datadriven environment [3] that lead to reshape organizational capabilities and generate economic value. These studies have explicitly explored the impact of big data analytics on facilitating decision making and enhancing organizational benefits.

However, BDA's value creation is a complex process which cannot be fully explained by a set of factors and regression-based methods, but instead involves the systemic and simultaneous arrangement of multiple elements. BDA alone does not unequivocally facilitate business value and the link between BDA and business value is not likely to be straightforward in terms of the multi-way interactions among various BDA capabilities and other organizational elements. To the best of our knowledge, as yet no previous studies have considered the complex interactions among BDA and the organizational elements driving BDA success to examine how organizational elements influence organizational performance in conjunction with BDA.

As neither prior studies are capable of explaining the full complexity of successful BDA implementations, this study seeks to explain the role of big data analytics in healthcare performance from a configuration theory perspective. Configuration theory argues that business value generation is a complex process [11] [12] [13] and is very difficult to portrait using regression-based methods [14]. As such, a systemic and simultaneous arrangement of multiple organizational elements interacting with BDA resources provides a more holistic view of how BDA can contribute to healthcare performance. This thus leads to our main research question:

What configurations of BDA capabilities, complementary organizational resources, and organizational capabilities lead to improved quality of care (i.e. low average excess readmission ratio) in health care?

To address this question, this study proposes a conceptual model with a configurational lens to show the complexity of big data analytics implementation. Specifically, we conceptualize BDA capability as a 
multi-dimensional construct that is shaped by a set of technological BDA resources (e.g., functionalities of BDA systems) and the skills and talents of analytical thinkers. We then go on to build on configuration theory and the literature on BDA by adding other organizational elements such as complementary organizational resources (i.e., an evidence-based decision making culture and data governance), and organizational capabilities (i.e., planned dynamic capabilities and improvisational capabilities) to consider the potential impact of BDA on the quality of care in health care.

\section{Theoretical Background}

\subsection{Brief Review: Path to Big Data Analytics Success}

To gain a better understanding of the role of BDA in creating business value, we review the studies aiming at exploring the impact of BDA on organizational performance, as summarized in Appendix 1.

Most of these studies have drawn on the resourcebased theory and IT capability literature to develop a set of BDA technical and human capabilities. Specifically, researchers have explored several BDAspecific capabilities that organizations could use to gain meaningful insights and reshape organizational performance, such as BDA infrastructure and functionalities [3] [10], and the capabilities of analytical personnel [15] [16].

Applying a broader view of IT resources from studies of the business value of IT to identify BDA's critical factors, several complementary organizational resources and organizational capabilities have been identified as intermediate variables that contribute to the creation of business value through the use of BDA, such as data-driven environment [3], data governance [4], information processing capabilities [3], dynamic capabilities [15] [16], and evidence-based decision making culture [17].

\subsection{Configuration theory}

Configuration theory emerging from organizational research and strategic management has the potential to fuel the next jump in the understanding of business value of big data analytics by complementing the potential incompleteness of both process theories and variance theories [18] [19]. Configuration is defined as "a specific combination of causal elements or conditions that generate an outcome of interest" [13]. Configuration theory allows researchers to understand a complex messy phenomenon by exploring its patterns and combinations of interconnected elements and reveal how its synergistic effects result in specific outcomes. Configuration theory also supports the concept of equifinality where the same outcome can be generated by one or more sets of configuration patterns [18] [20], which can provide new heuristic insights for big data analytics implementation by suggesting multiple strategic configurations from which managers can choose the optimal solution that fits their organizational context [21].

The application of configuration theory in the IS field is still in its infancy [21]. To the best of our knowledge, this study is among the first empirical studies examining business value of big data analytics from a configuration theory perspective although conceptual papers can be found in the literature. As business value generation is a complex process resulting from multi-way interactions among multiple elements, we posit that configuration theory is best suited for this study, and consequently use the analysis method designed for this type of study, Qualitative Comparative Analysis (QCA). QCA is a set-theoretic method that has been developed to properly capture the holistic nature of configurations theory and to determine how configurations that present the essential causal ingredients in sets are linked to specific outcomes [14]. QCA permits exploring the interplay of elements rather than showing the value of each factor contributing to the outcome.

\section{Research Model}

We employ Melville et al.'s [12] IT business value generation framework logics in exploring business value driven by big data analytics. Following Schryen [22], we intend to extend this framework from "business value should be rooted in the identification of IT resources" to "seeking for the best configuration of possible IT resources." This shift will show that various IT resources and complementary organizational resources and capabilities affect each other and can co-create business value. The purpose of this study is to fill the gap of understanding since the complexity interactions among IT resources and complementary organizational resources and capabilities remain unclear [22].

Our research model relied on the configuration theory to disentangle the complex interactions among the elements leading to high quality of care. Configuration theory is better suited for understanding patterns and combinations of factors and how they, as configurations, cause specific outcomes to occur in a certain context [18] [23] [24]. This configurational perspective provides the basis for our analysis of the causal paths that explain how, in health care context, the combination of big data analytics capabilities and 
other organizational elements may lead to superior quality of care. Specifically, we examine elements of big data analytics capabilities, complementary organizational resources, and organizational capabilities embedded in business process that can be combined into different configurations for high quality of care. Figure 1 illustrates the interactions among these three configuration elements of big data analytics through intersecting orbits as the holistic confluence that subsequently contributes to enhance quality of care in healthcare.

\subsection{The elements of big data analytics capabilities}

Big data analytics capability is defined as the ability to acquire, store, process and analyze large amount of data in various forms, and deliver meaningful information to users that allows them to discover business values and insights in a timely fashion [25]. We identified four generic categories of big data analytics capabilities from our review of the big data implementation cases.

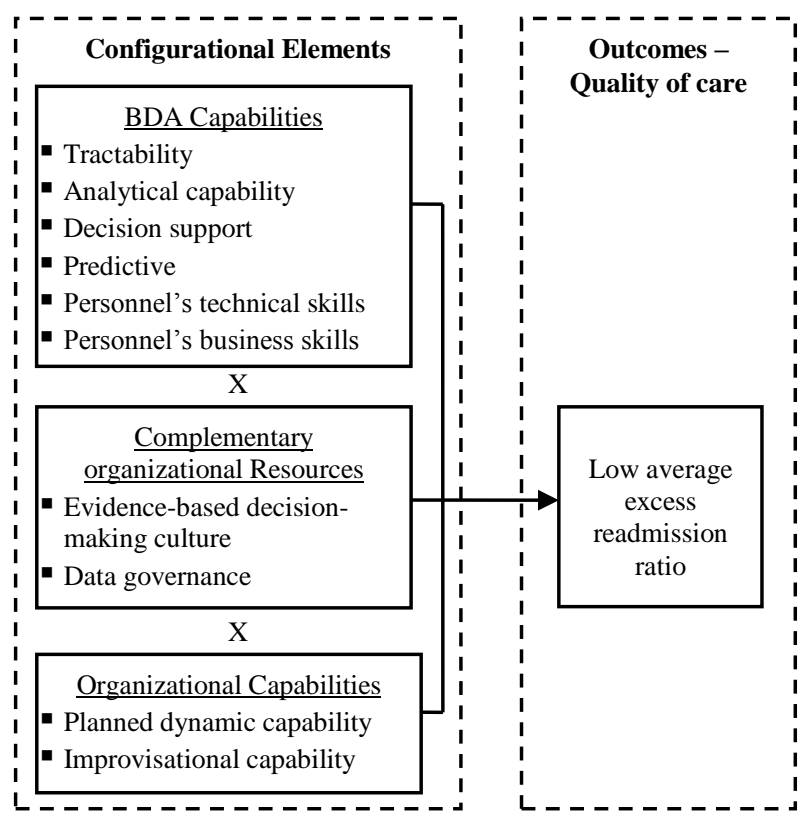

Figure 1. Research Model

Traceability is the ability to track output data from all the system's IT components throughout the organization's service units [26] [27]. The primary goal of traceability is to make data consistent, visible and easily accessible for analysis.

Analytical capability is defined as the ability to process data with an immense volume (from terabytes to exabytes), variety (from text to graph) and velocity (from batch to streaming) via unique data storage, management, analysis, and visualization technologies.
Analytical capabilities can be used to identify patterns of care and discover associations from massive healthcare records, thus providing a broader view for evidence-based clinical practice. In addition to identifying the patterns of care, analyzing unstructured health data is another key capability in a big data analytics system.

Decision support capability emphasizes the ability to produce reports about daily healthcare services to assist managers' decisions and actions [26]. In general, this capability yields sharable information and knowledge such as historical reporting, executive summaries, drill-down queries, statistical analyses, and time series comparisons. Such information can be utilized to provide a comprehensive view to support the implementation of evidence-based medicine, to detect advanced warnings for disease surveillance, and to develop personalized patient care.

Predictive capability is "the ability to apply diverse methods from statistical analysis, modeling, machine learning, and data mining to both structured and unstructured data to determine future outcomes" [28]. Predictive analysis makes it possible to cross reference current and historical data to generate context-aware recommendations that enable managers to make predictions about future events and trends. This capability relies on predictive analytical engines that incorporate a data warehouse, a predictive platform with predictive algorithms (e.g., decision trees, neural networks, and logistic regression), and a predictive interface that provides feedback and recommendations to users. Predictive capabilities can reduce degree of uncertainty, enable managers to make better decisions faster and hence support preventive care [6].

\subsection{Analytical personnel skills}

The role of analytical personnel is considered as a human IT resource in shaping the value of big data analytics [5]. Analytical personnel are defined as the organizational members who have an analytic mindset and help gain business insights using big data analytics tools [31]. Analytical personnel are a hybrid role that requires a broad combination of technical and soft skills from multidisciplinary knowledge domains. The skill sets for analytical personnel have been investigated in the literature. For example, based on the different levels of data analytical skills, Wilder and Ozgur [29] categorize analytical people as data scientist, data specialists, and big data analyst. Data scientist is defined as people who understand how to seek for answers to important questions from tsunami of unstructured information [31]. Data specialists are people who not only have a solid foundation in computer science, mathematics and management, but also understand how data is managed [29]. Business 
analysts (i.e., chief data officer) are key leaders in the organization responsible for establishing data quality governance and using data-driven insights to make sound decisions, identifying, exploiting business opportunities and addressing business problems [32].

\subsection{The elements of complementary organizational resources}

To implement big data analytics into practices, organizations must undergo adjustments or even dramatic changes regarding day-to-day operations, data policies, and organizational culture [29] [33]. Scholars have identified several key complementary organizational resources in the context of big data analytics such as enterprise-wise analytics orientation [4] and fact-based decision-making culture [4], [25]. In this study, we include two organizational resources, evidence-based decision making culture and data governance in our model for the configurations lead to better quality of care.

Organizational culture is defined as a set of collective values, beliefs, norms, and principles shared among organization members by defining appropriate behavior for various situations [34]. Organizational culture has long been recognized as an important role for organization performance by management and strategy scholars. This study focuses on a particular aspect of organizational culture from big data analytics perspective, namely evidence-based decision marking culture, defined as a culture of embracing evidencebased management and embedding evidence-based decision marking in the core values and processes of the organization [29]. Some scholars describe this concept as an information orientation culture that business executives have a heightened awareness of information and information management as they make decisions or formulate business strategies [35] while others view it as a data-driven culture, defined as "a pattern of behaviors and practices by a group of people who share a belief that having, understanding and using certain kinds of data and information plays a critical role in the success of their organization" [36]

Experts have realized that data governance practice is crucial for deriving business value [37]. Typically, data governance framework is comprised of master data management (MDM), data life cycle management, and data security and privacy management [27]. The key to successful data governance is not technology or methods; instead, it is about practices and people in the organization and their complex ownership of the data that big data analytics initiative will affect. Scholars describe this concept as an organization's data-driven environment that "is the organizational practices reflected by developing explicit data strategy and policy to guide analytic activities and designing its structure and process to enable and facilitate big data analytics activities" [3]. Data governance can also be viewed as a set of policies, a way of working, or a framework of optimizing the value of information in some sense to the decision makers involved [38]. In hospitals, for example, establishing rigorous data policies and data access control mechanisms for highly sensitive healthcare data can prevent security breaches and protect patient privacy.

\subsection{The elements of organizational capabilities}

Organizational capabilities are significant for business value creation in various contexts [39] [40]. In general, organizational capability is defined as the ability to adapt ongoing changes in the business processes and functional activities of the firm [41], while it is also described as "an organization's ability to create value in a unique way by utilizing resources" [42] from the RBV perspective. From a dynamic capability perspective, two types of distinctive organizational capabilities - planned dynamic capability and improvisational capability - have been identified from the core business processes for boosting business value [40]. Further, with a configurational lens, El Sawy et al. [13] highlight the role of IT systems in shaping these two capabilities. Planned dynamic capability is a firm's organizational ability to integrate, reconfigure, gain and renew resources to match rapidly-changing market environments [43] [44], and enhance a firm's agility [45]. Barreto [46] and Teece [47] view dynamic capability as the ability to sense and shape opportunities and threats, to seize market opportunities and to maintain competitiveness.

Improvisational capability is defined as an organization's learned ability to respond to unexpected environmental turbulences quickly by simultaneously forming and executing novel solutions by reconfiguring available resources [48]. Research from both strategic and organizational management fields has emphasized the importance of organizational improvisation to handle extreme competition, cope with changing circumstances, and pursue potential business opportunities (e.g. [49], [50]). Improvisational capability plays a crucial role in building organizational agility to react to market changes. Such "spontaneous" capabilities enable organizations to make effective and real-time decisions in response to turbulences without having to go through formal planning channel.

\section{Research Method}

For this study, healthcare industry was selected as our research context for two reasons: (1) big data 
analytics implementation in healthcare industries has lagged behind other industries such as retail and banking. Little is known about whether big data analytics adoption actually contributes to the growth of healthcare while other industries have obtained tremendous benefits driven by big data analytics, and (2) focusing on single industry can mitigate potential confounding effects due to industry nature and variation.

\subsection{Data collection}

An initial population set of 4668 senior IS executives (primarily Vice Presidents, CIOs, and IT directors) in US hospitals, listing the facility name, job title, phone number, and email address for each, was extracted from the Healthcare Information and Management Systems Society (HIMMS) database. After data cleaning to remove incomplete information and duplicates, 3307 senior IS executives remained. An online survey was specifically designed for this study. An information letter containing a description of the research purpose and an information privacy protection statement were distributed with the survey to potential participants via the Qualtrics survey platform. The first round of 3307 questionnaires resulted in 511 emails being blocked by their organizations' firewall and 1589 emails that were never opened; a gentle reminder was sent a week later. Of the 1207 invitations that were seen by potential respondents, 65 responses were returned, 63 of which were complete and usable for the data analysis, showing a response rate of $5.39 \%$. CMS data was downloaded from the Hospital Compare website (www.medicare.gov/hospitalcompare). This website provides information on how well hospitals provide healthcare service to their patients and allows them to compare performance metrics related to certain conditions. We extracted average excess readmission ratios from the CMS database to evaluate the care of quality as the outcome for this study. We were able to match CMS data to our survey data in 34 cases.

\subsection{Measurement}

We operationalize business value in hospital settings by using quality of care from Agarwal et al.'s [51] HIT impact framework. To assess the quality of care, we take advantage of the recently released Hospital Compare Data database in terms of Hospital Readmissions Reduction Program (HRRP) and Hospital Value-Based Purchasing (HVBP) Program based on applicable period of July 1, 2011 to June 30, 2014 from CMS. The average excess readmission ratio is used as one of the measures of quality of care [52]. A hospital's excess readmission ratio is a measure of a hospital's readmission performance compared to the national average for the hospital's set of patients with that applicable condition. While there are a variety of quality outcome measures that could be considered, we chose excess readmission ratio, as they are a reflection of the total process of care received [53]. The higher the ratio is, the worse the quality of care.

Besides the outcome variable, all other measurement items were adopted from the literature and modified to fit this study, as presented in Appendix 2. To assess the validity and reliability of measurements, a sample data set $(\mathrm{N}=63)$ collected for this study was analyzed using SmartPLS 2.0. We note that all of the reliability coefficients (Cronbach's alphas) are above 0.70 , showing that the measurements are reliable. Convergent validity was assessed by three criteria: (1) item loading, (2) composite reliability, and (3) average variance extracted (AVE). Loadings are all in acceptable ranges, the composite reliabilities scores range from 0.85 to 0.94. Each AVE is above 0.5, indicating that the latent construct can account for at least 50 percent of the variance in the items. Moreover, we employed two methods to assess discriminant validity. Each item loading in cross-loading table is much higher on its assigned construct than on the other constructs. The square root of the AVE is greater than all of the inter-construct correlations. Thus, our measurement demonstrates sufficient discriminant and convergent validities.

\subsection{Data Analysis Procedure using fuzzy-set Qualitative Comparative Analysis (fsQCA)}

In contrast to statistical regression-based methods, Qualitative Comparative Analysis (QCA) is based on set theory and logic and is designed to evaluate social systems characterized by causal complexity. QCA was developed in political science to evaluate case studies with too few cases for standard statistical analysis and where the available data are often qualitative or a combination of qualitative and quantitative [54], [55]. QCA is configurational because it allows investigators to identify combinations of configurations associated with an outcome of interest. We chose to apply the fuzzy-set approach because it offers an outlet that using the different degrees of membership in a set [20].

FsQCA allows a characteristic to have any continuous value from 0 to 1 , so that it is not "stated as either true or false, but as being possibly true to a certain degree" [56]. After case selection, a critical requirement in fsQCA analysis is to carefully convert data into measures of set membership using theoretical or substantive knowledge external to the empirical data - a process called calibration. We followed Ragin [20] in calibrating fuzzy-set memberships. For each calibration, we set thresholds based on industry common standards if available, extant theory or substantive knowledge. We used the direct method of 
calibration in the fsQCA software to transform the measures into set memberships [20], [57]. Survey items that are on Likert scale have somewhat built-in membership scores. All conditions were measured using a 1-7 scale so we calibrate them using 6, 4, 2 as the full membership, the crossover point, and the full non-membership anchors respectively.

We set up a "low average excess readmission ratio" set because the lower the ratio the better the quality. A national excess readmission ratio average was calculated by taking the mean of the rate from over 3,500 hospitals across the country as the industry standard. Full membership, cross-over, and full nonmembership are set as national average excess readmission ratio minus $1 \mathrm{SD}(0.92)$, the national average excess readmission ratio (0.99), and national average excess readmission ratio plus 1SD (1.10) respectively.

\section{Research Results}

In fsQCA, two central measurements provide parameters of fit: consistency and coverage [20], [24]. Consistency measures the degree to which a relation of necessity or sufficiency between a causal condition (or combination of conditions) and an outcome is met within a given data set [58]. It resembles the notion of significance in statistical models [59]. Consistency values range from " 0 " to " 1 ," with " 0 " indicating no consistency and " 1 " indicating perfect consistency. Each solution consistency "measures the degree to which membership in each solution term is a subset of the outcome" [20]. As shown in Table 1, all consistency scores for configurations are above the suggested cutoff value of .75 [60] which suggests that these models (solutions/recipes/configurations) are adequately specified. Once consistency has been established, coverage provides a measure of empirical relevance [60]. The analogous measure in statistical models would be $\mathrm{R}^{2}$, the explained variance contribution of a variable [59].

Five different configurations result in low average excess readmission ratio, meaning that five different paths could lead to this outcome. Analytical capability and decision support capability are evaluated as "necessary" conditions. A "necessary" condition is defined as that the outcome would not have happened without it. All the four big data analytics capabilities are either core or contributors in all solutions except that traceability is absent in solution 2. The two complementary organizational resources (evidencebased decision making culture, data governance only contribute to solutions 4 and 5 .

When a healthcare organization does not have highlevel resources such as evidence-based decision making culture, data governance and dynamic capabilities, it must have high level of analytical and decision support capabilities combined with traceability, personnel's technical and business skills, and improvisational capabilities to achieve high level of quality of care (Solution 1). When a healthcare organization lacks high level of traceability, personnel's skills, organizational resources and other capabilities (dynamic and improvisational capabilities), the combination of high level of analytical, decision support and predictive capabilities could lead it to low readmission rate (Solution 2). Another path to better quality of care would be the combination of mainly high levels of analytical and decision support capabilities and supportive roles of high level of traceability, predictive capability, analytics personnel's technical skills and dynamic capabilities, even without high levels of analytics personnel's business skills, decision making culture, data governance, and improvisational capabilities (Solution 3). Interestingly, the difference between solutions 3 and 4 is the "switching" of importance of data governance and dynamic capabilities. With all other elements equal, to get to better quality of care, a healthcare organization either builds its data governance or its dynamic capabilities. Solution 5 seems hard to achieve because it has all the causal elements present; however, it covers $5 \%$ of our cases uniquely, which in turn means that there are healthcare organizations that achieve high level of quality of care by building all the big data analytics capabilities with complementary organizational resources, dynamic and improvisational capabilities.

Table 1. Configurations for Low Average Excess Readmission Ratio ( $N=34$ cases)

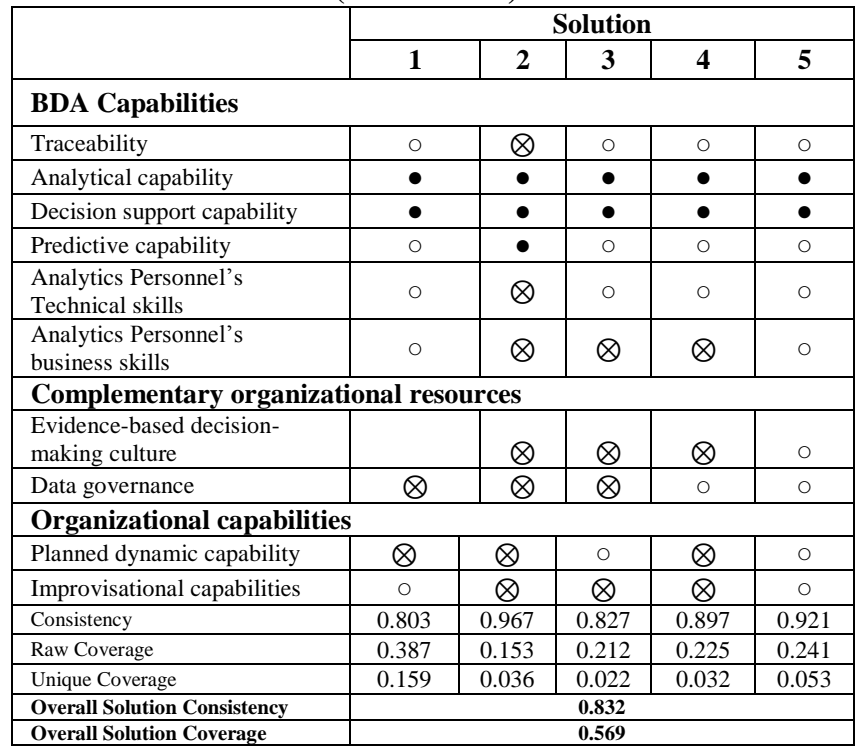


Note: $\bullet$ : Central core elements; ०: Peripheral elements; $\bigotimes$ : The absence of an element; Blank space: don't care

\section{Conclusion and Future Study}

Rather than examining the elements with linear associations, this study captures the complex interactions of the interdependencies among big data analytics capabilities and other organizational elements, and examines how different configurations cause improved quality of care in health care. In doing so, this research first contributes to theory by proposing a conceptual model with a holistic view that helps healthcare organizations scope their big data analytics initiatives. Secondly, based on empirical data, it identifies different configurations of conditions leading to higher quality of care in healthcare which extends and deepens the understanding of business value of big data analytics. Configurations found provide evidences for how different relational aspects interact with each other to create organizational performance in healthcare in different situations. Thirdly, our findings provide useful guidance for practitioners with regard to the management and configuration of big data analytics.

Contradictory to previous studies [17], the initial finding shows that evidence-based decision making culture is absent in most of solutions (except for solution 5). A possible explanation is that in a healthcare organization especially in a clinic when treating patients most physicians rely on their professional experiences in making decision instead on a system output that they are not familiar with or have not been trained to use it [25]. This requires further investigation. In the future, we plan to continue collecting data and examine other performance matrices.

\section{References}

[1] Raghupathi, W., and Raghupathi, V. "Big data analytics in healthcare: Promise and potential", Health Information Science and Systems, 2(1), 2014, pp. 1-10.

[2] Ward, M. J., Marsolo, K. A., and Froehle, C. M. "Applications of business analytics in healthcare", Business Horizons, 57(5), 2014, pp. 571-582.

[3] Cao, G., Duan, Y., and Li, G. "Linking business analytics to decision making effectiveness: A path model analysis", IEEE Transactions on Engineering Management, 62(3), 2015, pp. 384-395.

[4] Seddon, P.B., Constantinidis, D., and Dod, H. "How does business analytics contribute to business value?", In Proceeding of the 33rd International Conference on Information Systems, Orlando, Florida, USA, 2012.

[5] Tamm, T., Seddon, P., and Shanks, G. "Pathways to value from business analytics", In Proceeding of the 34th International Conference on Information Systems, Milan, Italy, 2013.
[6] Wang, Y., Kung, L., Wang, W. Y. C., and Cegielski, C. G. "An integrated big data analytics-enabled transformation model: Application to health care", Information \& Management, 2017 https://doi.org/10.1016/j.im.2017.04.001

[7] Sharma, R., Mithas, S., and Kankanhalli, A. "Transforming decision-making processes: A research agenda for understanding the impact of business analytics on organisations", European Journal of Information Systems, 23(4), 2014, pp. 433-441.

[8] Trkman, P., McCormack, K., De Oliveira, M. P. V., and Ladeira, M. B. "The impact of business analytics on supply chain performance", Decision Support Systems, 49(3), 2010, pp. 318-327.

[9] Wang, Y., and Byrd, T. A. "Business analyticsenabled decision making effectiveness through knowledge absorptive capacity in health care", Journal of Knowledge Management, 21(3), 2017, pp. 517-539.

[10] Wixom, B., Yen, B., and Relich, M. "Maximizing value from business analytics", MIS Quarterly Executive, 12(2), 2013, pp. 111-123.

[11] Bharadwaj, A. S. "A resource-based perspective on information technology capability and firm performance: An empirical investigation", MIS Quarterly, 24(1), 2000, pp. 169-196.

[12] Melville, N., Kraemer, K., and Gurbaxani, V. "Review - Information technology and organizational performance: An integrative model of IT business value", MIS Quarterly, 28(2), 2004, pp. 283-322.

[13] El Sawy, O. A., Malhotra, A., Park, Y., and Pavlou, P. A. "Seeking the configurations of digital ecodynamics: It takes three to tango", Information Systems Research, 21(4), 2010, 835-848.

[14] Fichman, R. G. "Going beyond the dominant paradigm for information technology innovation research: Emerging concepts and methods", Journal of the Association for Information Systems, 5(8), 2004, pp.314-355.

[15] Gupta, M., and George, J. F. "Toward the development of a big data analytics capability", Information \& Management, 53(8), 2016, pp.10491064.

[16] Wamba, S. F., Gunasekaran, A., Akter, S., Ren, S. J. F., Dubey, R., and Childe, S. J. "Big data analytics and firm performance: Effects of dynamic capabilities", Journal of Business Research, 70, 2017, pp. 356-365.

[17] Popovič, A., Hackney, R., Coelho, P. S., and Jaklič, J. "Towards business intelligence systems success: Effects of maturity and culture on analytical decision making", Decision Support Systems, 54(1), 2012, pp.729-739.

[18] Fiss, P. C. (2007). "A set-theoretic approach to organizational configurations", Academy of Management Review, 32(4), 2007, pp.1180-1198.

[19] Fiss, P. C., Cambré, B., and Marx, A. Configurational Theory and Methods in Organizational Research (Vol. 38). Bingley, UK: Emerald Group Publishing, 2013. 
[20] Ragin, C. C. Redesigning Social Inquiry: Fuzzy Sets and Beyond. Chicago: University of Chicago Press, 2008.

[21] Park, Y., and El Sawy, O. A. "The value of configurational approaches for studying digital business strategy", Research in the Sociology of Organizations, 38, 2013, pp. 205-224.

[22] Schryen, G. "Revisiting IS business value research: What we already know, what we still need to know, and how we can get there", European Journal of Information Systems, 22, 2013, 139-169.

[23] Meyer, A. D., Tsui, A. S., and Hinings, C. R. "Configurational approaches to organizational analysis", Academy of Management Journal, 36(6), 1993, pp.1175-1195.

[24] Ragin, C. C. User's Guide to Fuzzy-Set/Qualitative Comparative Analysis 2.0. Tucson, AZ: Department of Sociology, University of Arizona, 2008.

[25] Watson, H. J. "Tutorial: Big data analytics: Concepts, technologies, and applications", Communications of the Association for Information Systems, 34(article 65), 2014, pp.1247-1268.

[26] Wang, Y., Kung, L., and Byrd, T. A. "Big data analytics: Understanding its capabilities and potential benefits for healthcare organizations", Technological Forecasting and Social Change, 2016, dx.doi.org/10.1016/j.techfore.2015.12.019

[27] Wang, Y., Kung, L., Ting, C., and Byrd, T. A. Beyond a technical perspective: understanding big data capabilities in health care. In System Sciences (HICSS), 2015 48th Hawaii International Conference on (pp. 3044-3053). IEEE, 2015.

[28] Wessler, M. Big data analytics for dummies. New Jersey, John Wiley\&Sons, 2013.

[29] Davenport, T. H., Harris, J. G., and Morison, R. "Analytics at work: Smarter decisions, better results", Harvard Business Press, 2010.

[30] Wilder, C. R., and Ozgur, C. O. "Business analytics curriculum for undergraduate majors", INFORMS Transactions on Education, 15(2), 2015, pp.180-187.

[31] Davenport, T. H., and Patil, D. J. "Data scientist: The sexiest job of the 21st century", Harvard Business Review, 2012, pp. 70-76.

[32] Lee, Y., Madnick, S., Wang, R., Wang, F., and Zhang, H.. "A cubic framework for the chief data officer: Succeeding in a world of big data", MIS Quarterly Executive, 13(1), 2014, pp.1-13.

[33] LaValle, S., Lesser, E., Shockley, R., Hopkins, M. S., and Kruschwitz, N. "Big data, analytics and the path from insights to value", MIT Sloan Management Review, 52(2), 2011, pp.21-31.

[34] Ravasi, D., and Schultz, M. "Responding to organizational identity threats: Exploring the role of organizational culture", Academy of Management Journal, 49(3), 2006, pp.433-458.

[35] Kettinger, W., Zhang, C., and Marchand, D. A. "CIO and business executive leadership approaches to establishing company-wide information orientation", MIS Quarterly Executive, 10(4), 2011, pp.157-174.
[36] Kiron, D., Ferguson, R. B., and Prentice, P. K. "From value to vision: Reimagining the possible with data analytics", MIT Sloan Management Review, 54(3), 2013, pp.1-19.

[37] Khatri, V., and Brown, C. V. "Designing data governance", Communications of the ACM, 53(1), 2010, pp.148-152.

[38] Kooper, M. N., Maes, R., and Lindgreen, E. R. “On the governance of information: Introducing a new concept of governance to support the management of information", International Journal of Information Management, 31(3), 2011, pp.195-200.

[39] Pavlou, P. A., and El Sawy, O. A. "From IT leveraging competence to competitive advantage in turbulent environments: The case of new product development", Information Systems Research, 17(3), 2006, pp.198-227.

[40] Pavlou, P. A., and El Sawy, O. A. "The "third hand": IT-enabled competitive advantage in turbulence through improvisational capabilities", Information Systems Research, 21(3), 2010, pp.443-471.

[41] Luo, J., Fan, M., and Zhang, H. "Information technology and organizational capabilities: A longitudinal study of the apparel industry", Decision Support Systems, 53(1), 2012, pp.186-194.

[42] Wu, I. L., and Hu, Y. P. "Examining knowledge management enabled performance for hospital professionals: A dynamic capability view and the mediating role of process capability", Journal of the Association for Information Systems, 13(12), 2012, pp.976-999.

[43] Eisenhardt, K. M., and Martin, J. A. (2000). Dynamic capabilities: What are they? Strategic Management Journal, 21(10), pp.1105-1121.

[44] Teece, D., Pisano, G., and Shuen, A. "Dynamic capabilities and strategic management", Strategic Management Journal, 18(7), 1997, pp.509-533.

[45] Roberts, N., and Grover, V. "Leveraging information technology infrastructure to facilitate a firm's customer agility and competitive activity: An empirical investigation", Journal of Management Information Systems, 28(4), 2012, pp.231-270.

[46] Barreto, I. "Dynamic capabilities: A review of past research and an agenda for the future", Journal of Management, 36(1), 2010, pp.256-280.

[47] Teece, D. "Explicating dynamic capabilities: The nature and microfoundations of (sustainable) enterprise performance" Strategic Management Journal, 28(13), 2007, pp.1319-1350.

[48] El Sawy, O. A., and Pavlou, P. A. "IT-enabled business capabilities for turbulent environments", MIS Quarterly Executive, 7(3), 2008, pp.139-150.

[49] Hadida, A. L., and Tarvainen, W. "Organizational improvisation: A consolidating review and framework", International Journal of Management Reviews, 17(4), 2014, pp.437-459.

[50] Bergh, D. D., and Lim, E. N. K. "Learning how to restructure: Absorptive capacity and improvisational views of restructuring actions and performance", 
Strategic Management Journal, 29(6), 2008, pp.59361

[51] Agarwal, R., Gao, G., DesRoches, C., and Jha, A. K. "Research commentary - The digital transformation of healthcare: Current status and the road ahead", Information Systems Research, 21(4), 2010, pp.796809.

[52] Centers for Medicare \& Medicaid Services (CMS). System Requirements Specification Hospital Compare Downloadable Database Data Dictionary. Centers for Medicare \& Medicaid Services, 2014.

[53] Pye, J., Rai, A., and Baird, A. "Health Information Technology in U.S. Hospitals: How Much, How Fast?", In Proceeding of from the 2014 International Conference on Information Systems, Auckland, New Zealand, 2014.

[54] Ragin, C. C. The Comparative Method: Moving Beyond Qualitative and Quantitative Strategies. Berkeley: University of California Press, 1987.

[55] Rihoux, B., and Ragin, C. C. Configurational Comparative Methods: Qualitative Comparative Analysis (QCA) and Related Techniques, Thousand Oaks, CA: SAGE, 2009.

[56] Treadwell, W. A "Fuzzy set theory movement in the social sciences", Public Administration Review, 55(1), 1995, pp.91-98.

[57] Fiss, P. C. "Building better causal theories: A fuzzy set approach to typologies in organization research", Academy of Management Journal, 54(2), 2011, pp.393-420.

[58] Ragin, C. C., Drass, K. A., and Davey, S. Fuzzyset/qualitative comparative analysis 2.0. Tucson, Arizona: Department of Sociology, University of Arizona, 2006.

\section{Appendix 1}

Summary of the literature on BDA success models

\begin{tabular}{|c|c|c|c|c|c|}
\hline \multirow[b]{2}{*}{ Study } & \multirow[b]{2}{*}{ Methods } & \multicolumn{3}{|c|}{ Factors leading to organizational performance } & \multirow{2}{*}{$\begin{array}{c}\text { Organizational } \\
\text { performance triggered by } \\
\text { BDA }\end{array}$} \\
\hline & & $\begin{array}{c}\text { BDA } \\
\text { capabilities }\end{array}$ & $\begin{array}{c}\text { Complementary } \\
\text { organizational } \\
\text { resources }\end{array}$ & $\begin{array}{l}\text { Organization } \\
\text { capabilities }\end{array}$ & \\
\hline Akter et al. [62] & Empirical & $\mathrm{V}$ & & & Firm performance \\
\hline Cao et al. [3] & Empirical & $\mathrm{V}$ & $\mathrm{V}$ & V & $\begin{array}{l}\text { Decision-making } \\
\text { effectiveness }\end{array}$ \\
\hline Fink et al. [63] & $\begin{array}{l}\text { Mixed } \\
\text { methods }\end{array}$ & $\mathrm{V}$ & & V & $\begin{array}{l}\text { Operational and strategic } \\
\text { value }\end{array}$ \\
\hline Gupta and George [15] & Empirical & $\mathrm{V}$ & $\mathrm{V}$ & & $\begin{array}{l}\text { Market and operational } \\
\text { performance }\end{array}$ \\
\hline Popovič et al. [17] & Empirical & V & V & & $\begin{array}{l}\text { Effective use of information } \\
\text { in business process }\end{array}$ \\
\hline Seddon et al. [4] & Conceptual & V & $\mathrm{V}$ & V & $\begin{array}{l}\text { Organizational benefits } \\
\text { from analytics use }\end{array}$ \\
\hline Trkman et al. [8] & Empirical & $\mathrm{V}$ & & & Supply chain performance \\
\hline Wamba et al. [16] & Empirical & $\mathrm{V}$ & $\mathrm{V}$ & & Firm performance \\
\hline Wang and Hajli [64] & Case study & $\mathrm{V}$ & & & $\begin{array}{lr}\text { IT } & \text { Infrastructure, } \\
\text { operational, } & \text { organizational, } \\
\text { managerial, } & \text { strategic } \\
\text { benefits } & \end{array}$ \\
\hline
\end{tabular}

[59] Thiem, A. Set-relational fit and the formulation of transformational rules in fsQCA. COMPASSS Working Paper Series, http://www.compasss.org/wpseries/Thiem2010.pdf, 2010.

[60] Legewie, N. An introduction to applied data analysis with qualitative comparative analysis. Forum Qualitative Sozialforschung/Forum: Qualitative Social Research, 14(3), article 15, 2013.

[61] Ross, J. W., Beath, C. M., and Quaadgras, A. "You may not need big data after all", Harvard Business Review, 91(12), 2013, pp.90-98.

[62] Akter, S., Wamba, S. F., Gunasekaran, A., Dubey, R., and Childe, S. J. "How to improve firm performance using big data analytics capability and business strategy alignment?", International Journal of Production Economics, 182, 2016, pp. 113-131.

[63] Fink, L., Yogev, N., and Even, A. "Business intelligence and organizational learning: An empirical investigation of value creation processes", Information \& Management, 54(1), 2017, pp.38-56.

[64] Wang, Y., and Hajli, N. "Exploring the path to big data analytics success in healthcare", Journal of Business Research, 70, 2017, 287-299.

[65] Kung, L., Kung, H. J., Jones-Farmer, A., and Wang, Y. "Managing big data for firm performance: A configurational approach", In Proceeding of 2015 Americas Conference on Information Systems (AMCIS), Puerto Rico, 2015.

[66] Cegielski, C. G., and Jones-Farmer, L. A. "Knowledge, Skills, and Abilities for Entry - Level Business Analytics Positions: A Multi-Method Study", Decision Sciences Journal of Innovative Education, 14(1), 2016, pp.91-118. 


\begin{tabular}{|l|l|c|c|c|l|}
\hline Wang and Byrd [9] & Empirical & V & V & & $\begin{array}{l}\text { Decision-making } \\
\text { effectiveness }\end{array}$ \\
\hline Wixom et al. [10] & Case study & V & & $\begin{array}{l}\text { Transactional, } \\
\text { informational, and strategic } \\
\text { value }\end{array}$ \\
\hline Kung et al. [65] & Empirical & V & & V & Firm performance \\
\hline
\end{tabular}

\section{Appendix 2 Measurement and Items}

Big data Analytics Capabilities

Our big data analytics tools have the ability to......

Tractability (Wang et al. [6])

- Integrate seamlessly clinical data across multiple departments in near real time or real time

- Track medical events based on the rules that built on hospital claims

- Search clinical databases for all data related to patients

Analytical capability (Wang et al. [6])

- Analyze large amounts of clinical data to understand the past and current state for specific target variables

- Explore the causes of medical events from clinical data

- Support real-time processing of multiple clinical data streams

Decision support capability (Wang et al. [6])

- Generate clinical summary in real time or near real time and present in visual dashboards

- Provide system outputs for role-based decisionmaking

Predictive analytics capability (Wang et al. [6])

- Discover patterns among specific variables of interest across departments

- Analyze data from different sources and use the results to predict future trends

- Provide actionable insights from clinical data in a format readily understood by healthcare providers

Analytics personnel technical skills (Cegielski and Jones-Farmer [66])

Our analytics personnel have the ability to......

- Integrate analyses from multiple sources into a business solution

- Use data visualization/graphical tools to interpret data

- Frame a business problem or question analytically

- Solve pre-framed business problems or questions analytically

Analytics personnel business skills (Cegielski and Jones-Farmer [66])

Our analytics personnel have

- Ability to be an independent learner

- Organizational skills

- Healthcare knowledge

Evidence-based decision making culture (Popovič et al. [17])
- Our hospital usually uses evidence-based insights for the creation of new service/product.

- Our hospital is open to new ideas and approaches that challenge current or future projects on the basis of new insights.

- Our hospital allows incorporating available information within any decision-making process.

Data governance (Khatri and Brown, [37])

- Data principle (clarifying the role of data as an asset)

- Data quality (establishing the requirements of intended use of data)

- Metadata (establishing the semantics of data so that it is interpretable by the users)

- Data access (specifying access requirement of data)

- Data lifecycle (determining the definition, production, retention and retirement of data)

Planned dynamic capabilities (Pavlou and El Sawy, [40])

- Our hospital frequently generates, disseminate, and respond to market intelligence about customer needs.

- Our hospital has adequate routines to acquire, assimilate, transform, and exploit existing resources to generate new knowledge.

- Our hospital is effective in managing dependencies among resources and tasks to synchronize activities.

- Our hospital effectively integrates disparate employees' inputs through heedful contribution, representation, and interrelation into our group.

Improvisational capabilities (Pavlou and El Sawy, [40])

- Our hospital is successful in figuring out our actions as we go along.

- Our hospital effectively improvises in carrying out our activities.

- Our hospital could spontaneously readjust our activities according to competitive environments. 